\title{
Adenovirus-mediated overexpression of cystic fibrosis transmembrane conductance regulator enhances invasiveness and motility of serous ovarian cancer cells
}

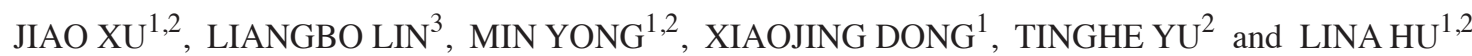 \\ ${ }^{1}$ Department of Obsterics and Gynecology; ${ }^{2}$ Laboratory of Obsterics and Gynecology, \\ The Second Affiliated Hospital of Chongqing Medical University, Chongqing 400010; \\ ${ }^{3}$ Stem Cell Biology and Therapy Laboratory, The Children's Hospital of Chongqing Medical University, \\ Chongqing 400014, P.R. China
}

Received November 28, 2014; Accepted August 25, 2015

DOI: $10.3892 / \mathrm{mmr} .2015 .4509$

\begin{abstract}
The cystic fibrosis transmembrane conductance regulator (CFTR) belongs to the adenosine triphosphate-binding cassette transporter family, members of which are involved in several types of cancer. Previous studies by our group reported that CFTR was highly expressed in serous ovarian cancer (SOC) tissues, and that knockdown of CFTR suppressed the proliferation of ovarian cancer in vitro and in vivo. Thus, the aim of the present study was to construct a recombinant adenoviral vector for the expression of the human CFTR gene in order to study the role of CFTR overexpression in the malignant invasion and migration of SOC cells in vitro. The present study then focused on the mechanisms of the role of CFTR in the migratory and invasive malignant properties of SOC cells. The CFTR gene was inserted into an adenoviral vector by using the AdEasy system in order to obtain the Ad-CFTR overexpression vector, which was used to transfect the A2780 SOC cell line. Reverse-transcription polymerase chain reaction, western blot analysis and immunofluorescence were performed to detect the expression and localization of CFTR. Cell invasion and motility of the transfected cells compared with those of control cells were observed using Transwell and wound healing assays. A $~ 4,700$ bp fragment of the CFTR gene was confirmed to be correctly cloned in the adenoviral vector and amplification of Ad-CFTR was observed in HEK293 cells during package. After 48 h of transfection with Ad-CFTR, 90\% of A2780 cells were red fluorescence protein-positive. Immunofluorescence showed that following
\end{abstract}

Correspondence to: Professor Lina Hu, Department of Obsterics and Gynecology, The Second Affiliated Hospital of Chongqing Medical University, 76 Linjiang Road, Chongqing 400010, P.R. China E-mail: cqhulina@hotmail.com

Key words: adenoviral vector, cystic fibrosis transmembrane conductance regulator, homologous recombination, serous ovarian cancer, tumor progression transfection, CFTR expression was increased and CFTR was located in the cell membrane and cytoplasm. CFTR overexpression was shown to enhance the invasion and motility of A2780 cells in vitro. Furthermore, the effects of CFTR overexpression on the activation c-Src signaling were observed by western blot analysis. CFTR overexpressing cells showed the lowest activity of phospho-Src (Tyr530), suggesting that CFTR may affect the activation of c-Src signaling. The results of the present study demonstrated that adenovirus-mediated CFTR overexpression enhanced cell invasion and motility of SOC cells in vitro. Furthermore, CFTR may be critical for the activation of c-Src signaling.

\section{Introduction}

Ovarian cancer is the fifth most common cause of cancer-associated mortality in females worldwide according to the global cancer statistics for 2013 (1). The most common histological sub-type of ovarian cancer is serous ovarian cancer (SOC). It is the most lethal type of gynecological cancer and, in spite of advances in the detection of SOC and cytotoxic therapies, has a five-year survival rate of $<30 \%$ (2). The main reason for the high mortality of patients with SOC may be due to the fact that $>70 \%$ of patients are diagnosed at an advanced stage of the disease. Although a large number of biomarkers with potential use for diagnosis and drug development have been reported (3-5), the detailed underlying mechanisms of the development of metastasis of this neoplasia have remained elusive.

Numerous studies have indicated that the adenosine triphosphate (ATP)-binding cassette (ABC) transporter family, which is an ancient family of transmembrane proteins, is frequently upregulated in metastatic cancer types and leads to chemotherapeutic resistance (6). The ABCC sub-family of $\mathrm{ABC}$ transporters includes cystic fibrosis transmembrane conductance regulator (CFTR) and the multidrug resistance-associated proteins, which are active drug exporters (7). CFTR, a $\sim 170-\mathrm{kDa}$ glycosylated protein, is known to be a cyclid adenosine monophosphate-dependent chloride anion-conducting channel (8). CFTR is expressed in epithelial 
cells of the human female reproductive tract (9) and overexpression of CFTR is associated with human cervical cancer as well as its progression and prognosis (10).

Previous studies by our group reported high expression of CFTR in SOC tissues, while knockdown of CFTR suppressed the proliferation of ovarian cancer in vitro and in vivo (11). Thus, it was hypothesized that overexpression of CFTR may be involved in the progression of SOC. In order to verify this hypothesis, the present study constructed an adenoviral vector for the forced overexpression of the CFTR gene in target cells. SOC cells were then transfected with this adenovirus to induce CFTR overexpression, and the effects of CFTR on cancer progression were studied using Transwell and wound healing assays. Furthermore, the present study assessed the mechanisms of the effects of CFTR on the migratory and invasive malignant properties of SOC cells.

\section{Materials and methods}

Materials. Shuttle plasmid pAdTrace-TOX, E1/E3-deleted replication-defective adenoviral backbone plasmid pAdEasy1 and the BJ5183 Escherichia coli (E. coli) strain were kindly provided by Dr Tongchuan He (Molecular Oncology Laboratory, Department of Surgery, The University of Chicago Medical Center, Chicago, IL, USA). HEK293 cells were obtained from the American Type Culture Collection (Manassas, VA, USA). The pcDNA3.1(+) vector containing the full-length purified human CFTR gene was kindly provided by Dr WenMing Xu (Key Laboratory of Obstetric, Gynecologic and Pediatric Diseases and Birth Defects of Ministry of Education, West China Second University Hospital, Sichuan University, Chengdu, China). DH5 $\alpha$ and A2780 cells were purchased from the China Center for Type Culture Collection (Wuhan, China). Cells used at passage 3-5. Dulbecco's modified Eagle's medium (DMEM) was purchased from Hyclone. Plasmid Mini kit (cat no. DP103) was purchased from Tiangen Biotek Inc. (Beijing, China). A Gel Extraction kit (cat no. PD1601) was purchased from BioTeke Corp. (Beijing, China). Restriction enzyme and T4 DNA Ligase were obtained from Thermo Fisher Scientific (Waltham, MA, USA).

pAdTrace-CFTR-TOX construction. The adenovirus was constructed using the AdEasy system (kindly provided by Dr Tongchuan He) (12). The human CFTR gene was digested with EcoRV, SalI and KpnI (Thermo Fisher Scientific) from pcDNA3.1-CFTR, which was directionally cloned into the shuttle plasmid pAdTrace-TOX vector containing a red fluorescent protein (RFP) reporter gene, cytomegalovirus (CMV) promoters, as well as PmeI and PacI sites. The ligation of purified CFTR fragment to pAdTrace-TOX at the molar ratios of 1:1, 2:1 and 3:1 was achieved via incubation with T4 DNA ligase (Thermo Fisher Scientific) at $22^{\circ} \mathrm{C}$ for $1 \mathrm{~h}$. The plasmid was extracted from clones resistant to $50 \mu \mathrm{g} / \mathrm{ml} \mathrm{kanamycin}$ (KeyGen Biotech, Nanjing, China) with sterile double-distilled water and screened by digestion with restriction endonuclease EcoRV and KpnI (Thermo Fisher Scientific).

Construction of recombinant adenoviral plasmids by homologous recombination in E. coli BJ5183 cells. The correct recombinant pAdTrace-TOX-CFTR plasmid, which was linearized by PmeI, was transfected into BJ5183 E. coli cells together with the adenoviral backbone vector pAdEasy-1. The transformed cells were plated on LB agar medium plates (Guduo Biotechnology, Shanghai, China) containing $100 \mu \mathrm{g} / \mathrm{ml}$ ampicillin and incubated at $37^{\circ} \mathrm{C}$ overnight. Plasmids extracted from small colonies using the Plasmid Mini kit (cat no. DP103; Tiangen Biotek Inc.) were separated by $0.8 \%$ agarose gel electrophoresis and examined by digestion with restriction endonuclease HindIII (Thermo Fisher Scientific). Gene Ruler $^{\mathrm{TM}}$ 1-kb DNA ladder (cat no. SM1333; Thermo Fisher Scientific) was used as a marker. The recombinant adenoviral plasmid was named as pAd-CFTR and transformed into E. coli DH5 $\alpha$ cells (cat. no. CB101-02; Tiangen Biotek Inc.) for amplification.

Transfection of recombinant adenovirus into HEK293 cells. HEK293 cells were cultured in DMEM supplemented with $10 \%$ fetal bovine serum (FBS; Invitrogen Life Technologies) at $37^{\circ} \mathrm{C}$ in a humidified atmosphere containing $5 \% \mathrm{CO}_{2}$. pAd-CFTR plasmid was linearized using PacI and transfected into HEK293 cells using Polyjet ${ }^{\mathrm{TM}}$ transfection reagent (SignaGen Laboratories, Gaithersburg, MD, USA) to package the recombinant adenoviral vector containing the CFTR gene according to the manufacturer's instructions. After 24 and $48 \mathrm{~h}$ of culture, RFP expression was observed by fluorescence microscopy (T-P2; Nikon, Tokyo, Japan). At 10-14 days after transfection, the cells were collected and subjected to three cycles of freezing at $-80^{\circ} \mathrm{C}$, thawing in a $37^{\circ} \mathrm{C}$-water bath and vigorous vortexing. The recombinant adenovirus containing the CFTR gene was then collected.

Adenovirus-mediated overexpression of CFTR in A2780 cells. A2780 cells were seeded into six-well culture plates at $50-60 \%$ confluence and the adenovirus was added to the medium. After $48 \mathrm{~h}$ of transfection, RFP-positive cells were observed under a microscope (T-P2; Nikon). Cells $\left(1 \times 10^{6}\right)$ were collected and suspended in $1 \mathrm{ml} 1 \%$ bovine serum albumin (BSA; KeyGen Biotech) in phosphate-buffered saline. The ratio of RFP-positive to total cells was acquired by flow cytometry using a FACSCanto ${ }^{\mathrm{TM}}$ II system and Cell Quest Pro software (BD Biosciences, Franklin Lakes, NJ, USA). Reverse-transcription quantitative polymerase chain reaction (RT-qPCR), western blot analysis and immunofluorescence were performed to detect the expression of CFTR. An empty adenovirus (Ad-null) was used as an adenoviral control.

$R T-q P C R$. After 48 h of culture, total RNA was isolated from cells using TRIzol reagent (Invitrogen Life Technologies, Carlsbad, CA, USA) according to the manufacturer's instructions. $1 \mu \mathrm{g}$ total RNA was used for reverse transcription in a $20-\mu 1$ reaction volume using the PrimeScript $\mathrm{RT}$ reagent kit (Takara Bio Inc., Otsu, Japan). The following primers (all from Sangon Biotech, Shanghai, China) were used: Human CFTR forward, 5'-TGCCCTTCGGCGATGTTT-3' and reverse, 5'-GCGATAGAGCGTTCCTCCTTG-3' (product length, 147 bp); GAPDH forward, 5'-CAGCGACACCCA CTCCTC-3' and reverse, 5'-TGAGGTCCACCACCCTGT-3' (product length, 122 bp). qPCR was performed using a CFX96 real-time system (Bio-Rad Laboratories, Inc., Hercules, CA, 
A

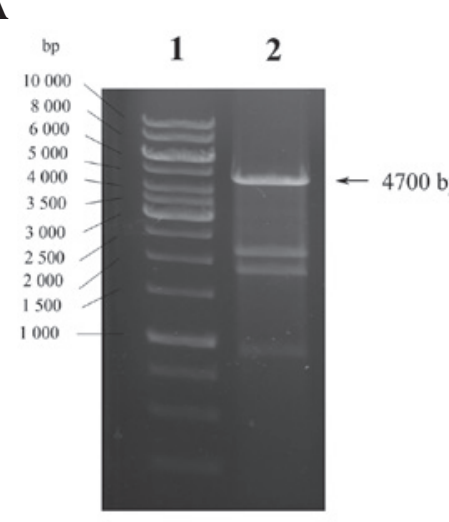

B

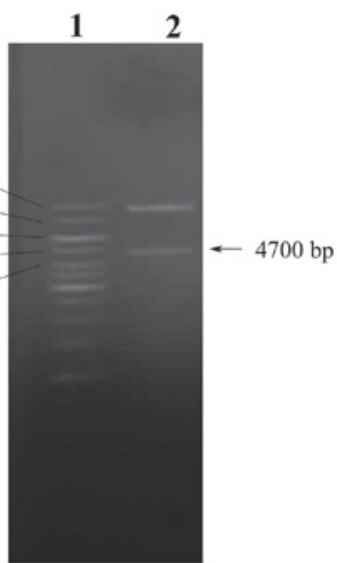

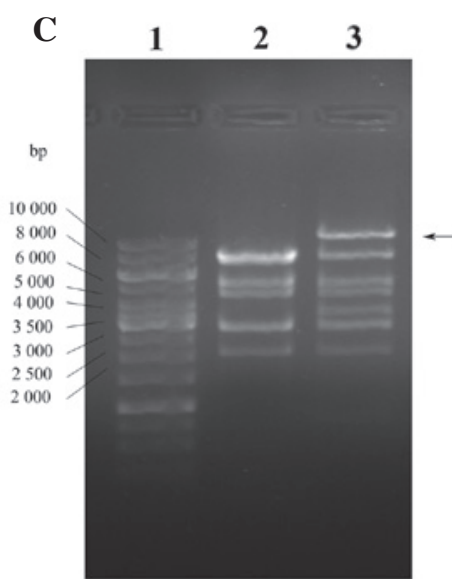

Figure 1. Construction and identification of Ad-CFTR vector. (A) Identification of pcDNA3.1-CFTR plasmid by enzyme digestion. Lanes: 1, Gene Ruler ${ }^{\text {TM }}$ 1-kb DNA Ladder; 2, pcDNA3.1-CFTR digested by EcoRV, SalI and KpnI enzymes to liberate the CFTR gene (4.7 kb). (B) Identification of recombinant pAdTrace-TOX-CFTR plasmid by enzyme digestion. Lanes: 1, Gene Ruler ${ }^{\mathrm{TM}} 1 \mathrm{~kb}$ DNA Ladder; 2, pAdTrace-TOX-CFTR plasmid digested by KpnI and $E c o R V$ enzymes, indicating the human CFTR gene was cloned into the shuttle plasmid. (C) Identification of recombinant pAd-CFTR by enzyme digestion. Lanes: 1, Gene Ruler ${ }^{\mathrm{TM}} 1 \mathrm{~kb}$ DNA Ladder; 2, pAdEasy-1 bone vector digestion with HindIII as the negative control; 3, digestion products of pAd-CFTR by HindIII. The largest fragment of pAd-CFTR was larger than the pAdEasy-1 bone vector ( 4,700 kb), indicating the successful construction of the recombinant adenoviral expression vector. CFTR, cystic fibrosis transmembrane conductance regulator; Ad, adenovirus.

USA). PCR was performed at $95^{\circ} \mathrm{C}$ for 3 min followed by 40 cycles at $95^{\circ} \mathrm{C}$ for $3 \mathrm{sec}$ and $60^{\circ} \mathrm{C}$ for $20 \mathrm{sec}$. Relative quantification of gene expression was performed using the $2^{-\Delta \Delta C T}$ method (13). Real-time PCR analysis was performed in at least three independent experiments.

Western blot analysis. Following transfection, cells were collected and lysed with Lysis Buffer (Beyotime Institute of Biotechnology, Haimen, China) containing phenylmethanesulfonylfluoride. The protein concentration was measured using a bicinchoninic acid protein assay kit (Beyotime Institute of Biotechnology). 50-100 $\mu \mathrm{g}$ protein per lane was separated by $8 \%$ SDS-PAGE and transferred onto a polyvinylidene difluoride membrane (Millipore Corp., Billerica, MA, USA). After blocking with $5 \%$ skimmed milk at $37^{\circ} \mathrm{C}$ for $1 \mathrm{~h}$, the membranes were incubated in mouse monoclonal anti-human CFTR antibody (diluted at 1:1,000; cat no. ab2784; Abcam, Cambridge, UK), and rabbit polyclonal GAPDH antibody (sc-25778; Santa Cruz Biotechnology, Inc., Dallas, TX, USA) at $4^{\circ} \mathrm{C}$ overnight. After washing with Tris-buffered saline containing Tween 20, membranes were incubated with the appropriate horseradish peroxidase-conjugated secondary antibodies (goat anti-mouse IgG-HRP; cat. no. sc-2005; Santa Cruz Biotechnology, Inc.) for $1 \mathrm{~h}$ at room temperature. Proteins were visualized using an enhanced chemiluminescence kit (cat no. 34080; GE Healthcare, Little Chalfont, UK) and images were captured using the ChemiDoc XRS system (Bio-Rad Laboratories, Inc.).

Immunofluorescence staining. Transfected cells were fixed with methanol at $-20^{\circ} \mathrm{C}$ for 15 min and blocked with $1 \%$ BSA at room temperature for $30 \mathrm{~min}$, followed by incubation with CFTR primary antibody (diluted at 1:100; cat no. ab2784; Abcam) at $4^{\circ} \mathrm{C}$ overnight. After washing with Tris-buffered saline containing Tween-20 twice, cells were incubated with fluorescein isothiocyanate-conjugated secondary antibody (Zhongshan Golden Bridge Inc., Zhongshan, China) at room temperature for $1 \mathrm{~h}$ in the dark. DAPI (Invitrogen Life Technologies, Inc.) was used to stain the nuclei. The presence of the proteins was examined under an inverted fluorescence microscope (T-P2; Nikon). Untreated SOC cells stained with mouse isotype-specific immunoglobulin M (Rockland Immunochemicals, Limerick, PA, USA) were used as negative controls.

Cell invasion assay. In the present study, cell invasion was determined by a Transwell assay using the Cell Invasion Assay kit ( $8 \mu \mathrm{m}$ pore size; Cell Biolabs, Inc., San Diego, CA, USA) according to the manufacturer's instructions. Approximately $1 \times 10^{5}$ cells were seeded into the upper chamber of the Transwell insert with serum-free media, and the bottom chambers were filled with medium containing $10 \% \mathrm{FBS}$ as a chemoattractant. After incubation for $24 \mathrm{~h}$ in a humidified incubator at $37^{\circ} \mathrm{C}$, cells on the upper side of the Transwell membrane were wiped off. Any cells that had transgressed through the membrane and were located at the lower side of the insert were fixed with $4 \%$ formaldehyde and stained in $0.5 \%$ crystal violet solution (KeyGen Biotech). Images of the cells were captured using a light microscope (TS100; Nikon, Tokyo, Japan) under x100 magnification and cells in random fields were counted. The procedure was repeated independently three times with triplicate inserts in each group.

Wound healing assay. Cell migration was evaluated in vitro using the scratch wound healing assay. In brief, $\sim 5 \times 10^{5}$ cells were seeded onto a six-well plate and grown to a confluent monolayer. A line-shaped wound of the surface of confluent cells was generated using a pipette tip. The cells were washed with growth medium to remove any cell debris, and to smooth the edges of the scratch wound. The cells were then incubated at $37^{\circ} \mathrm{C}$; cellular migration was evaluated and images were captured using a phase-contrast microscope. The width of the same area of the wound was observed at 0 and $24 \mathrm{~h}$, and the 
A

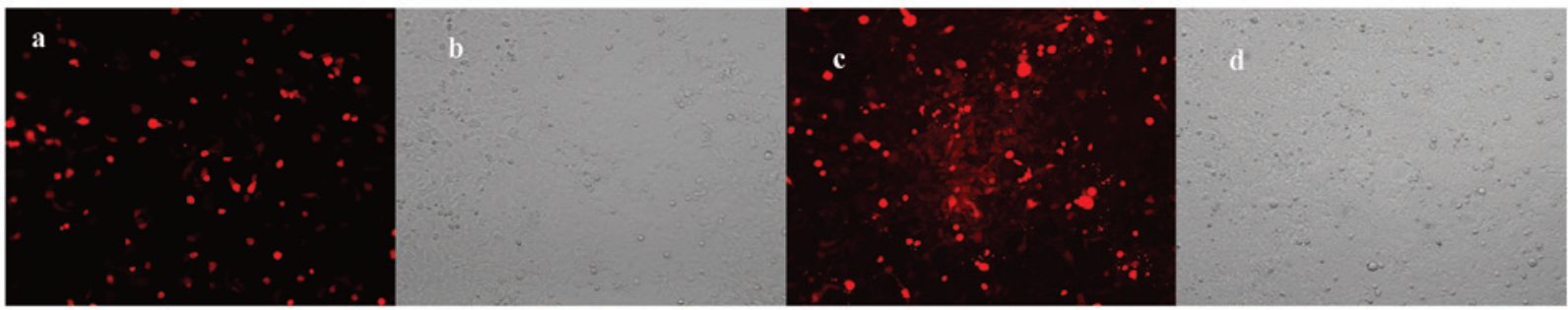

B
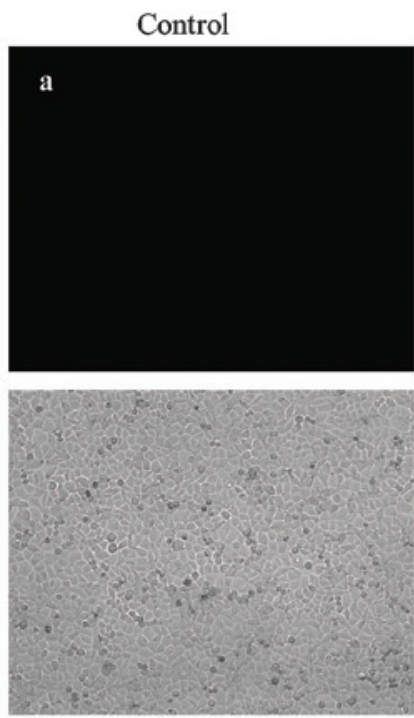
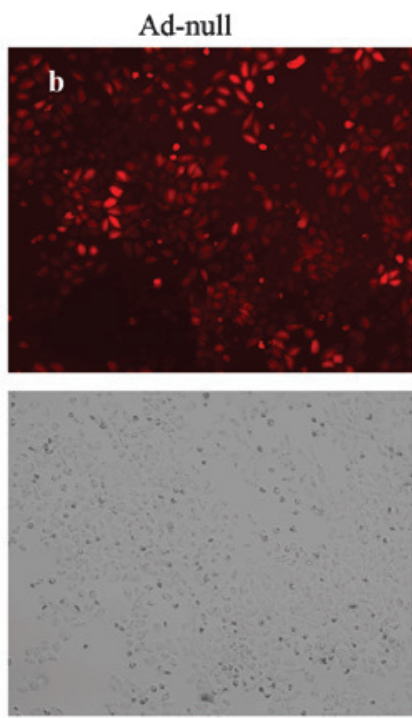

Ad-CFTR
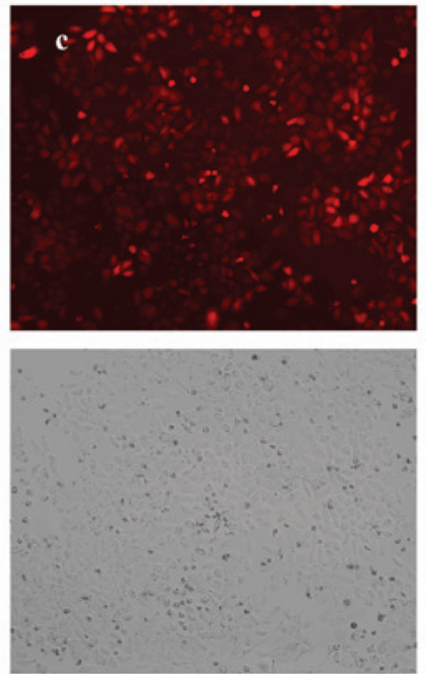

Figure 2. RFP expression was observed in HEK293 and A2780 cells. (A) Packaging of Ad-CFTR in HEK293 cells. After digestion by PacI and purification, pAd-CFTR was transfected into HEK293 cells. (a and b) pAd-CFTR-transfected HEK293 cells at 1 day. (c and d) Amplification of adenovirus Ad-CFTR was observed in HEK293 cells at 10 days of transfection. (B) RFP expression was observed in A2780 cells. (a) Untransfected A2780 cells as control, (b) d-null-infected A2780 cells, (c) Ad-CFTR-infected A2780 cells. Fluorescence and transmitted light microscopy images are shown (magnification, x100). RFP, red fluorescence protein; CFTR, cystic fibrosis transmembrane conductance regulator; Ad, adenovirus.

A
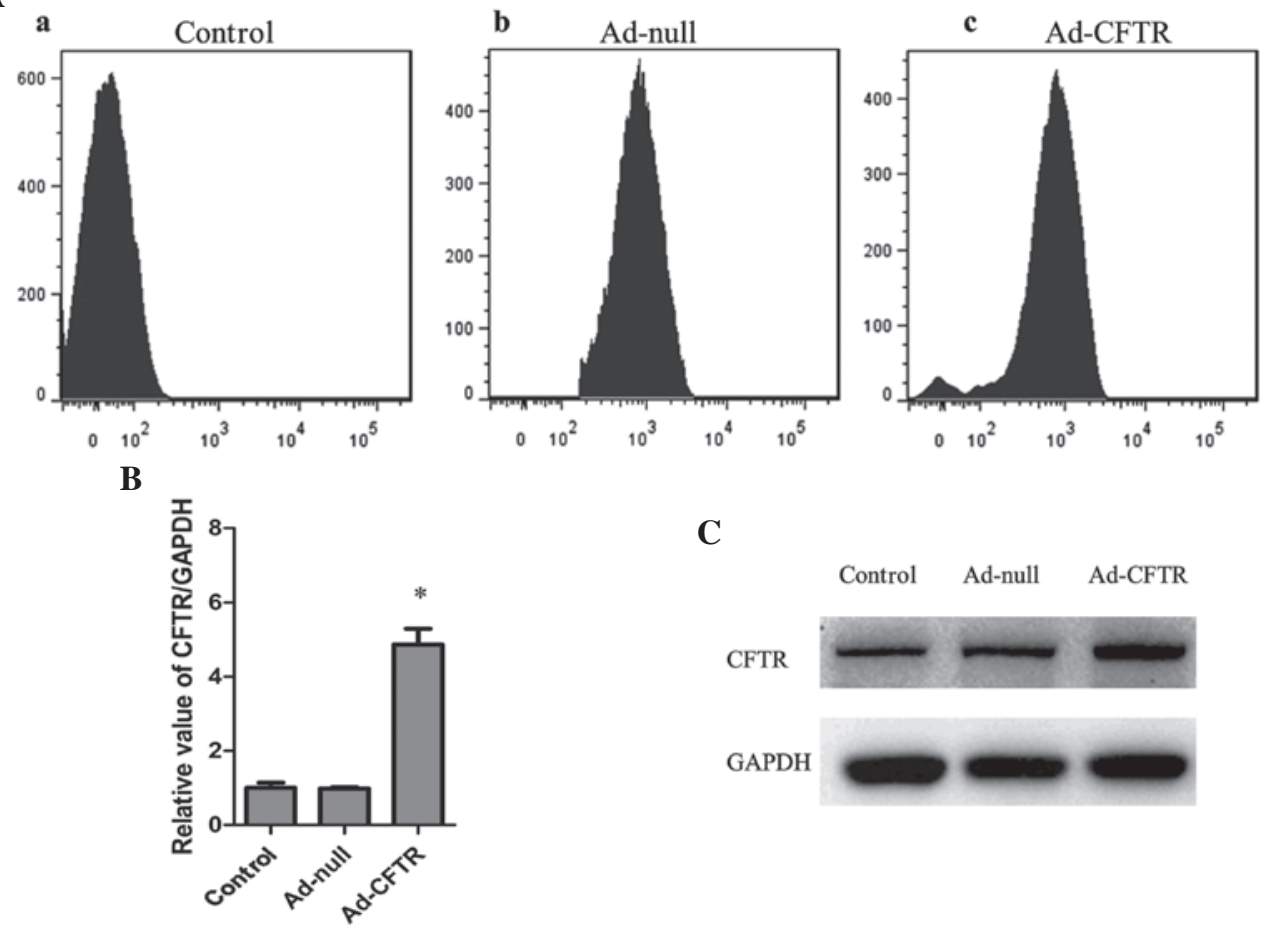

C

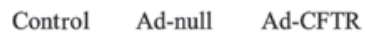

CFTR

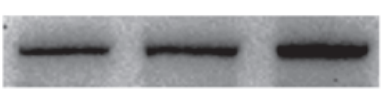

GAPDH

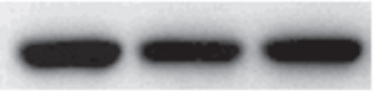

Figure 3. Infected efficacy of Ad-CFTR in A2780 cells. (A) Observation of red fluorescence protein by flow cytometry. (a) Uninfected A2780 cells as control, (b) Ad-null-infected A2780 cells, (c) Ad-CFTR-infected A2780 cells. (B) Expression of CFTR was analyzed by reverse-transcription quantitative polymerase chain reaction with normalization to GAPDH. Values are expressed as the mean \pm standard deviation. " $\mathrm{P}<0.05$ vs. control. (C) Western blot analysis of CFTR with GAPDH used as an equal loading control. CFTR, cystic fibrosis transmembrane conductance regulator; Ad, adenovirus. 
A

CFTR
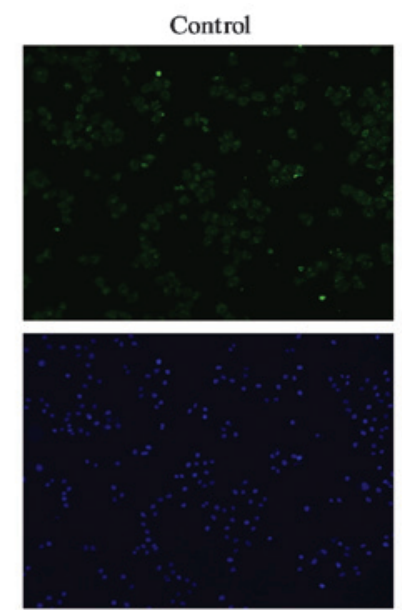

B
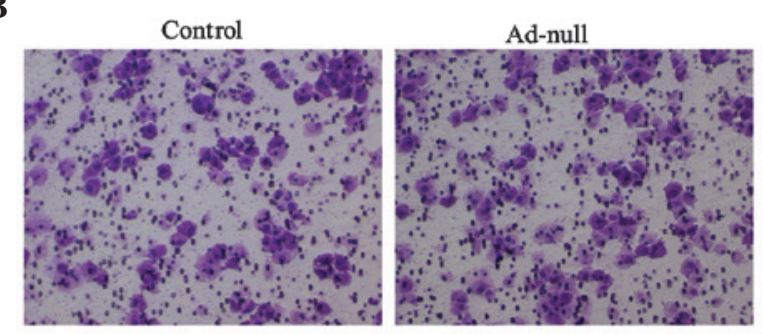

D

$0 \mathrm{~h}$

Control
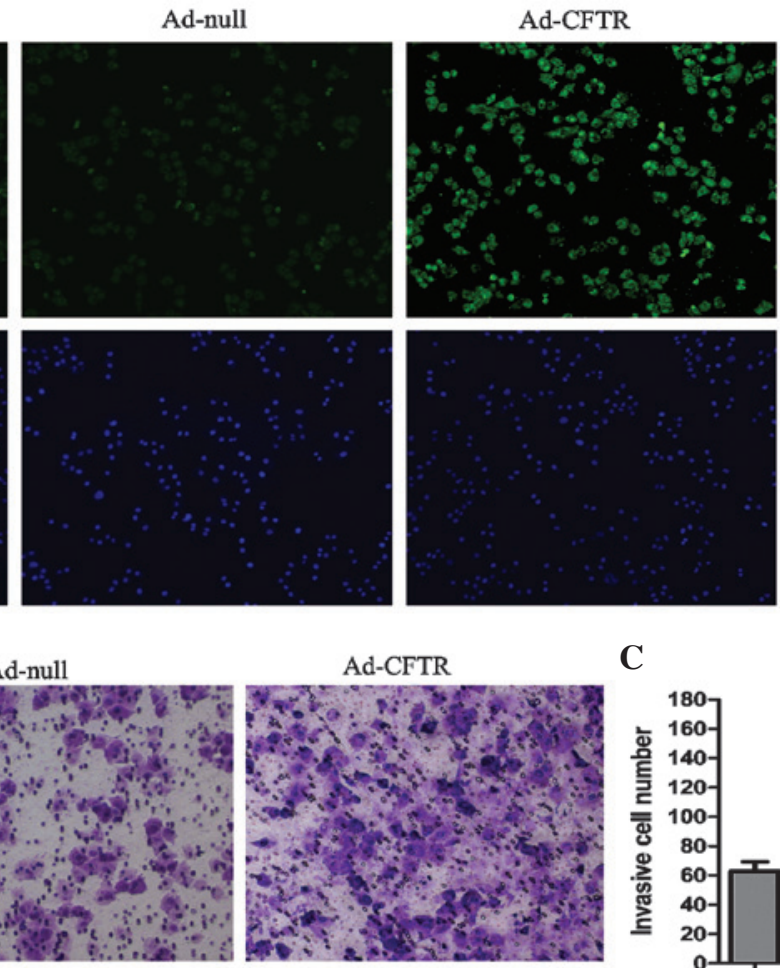

C

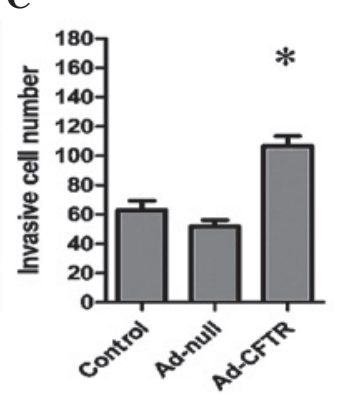

E

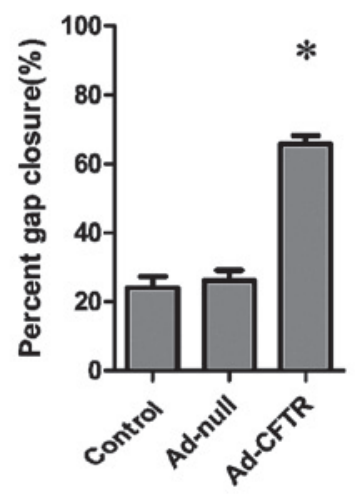

Figure 4. Localization and expression of CFTR in Ad-CFTR-transfected A2780 cells; overexpression of CFTR enhances the invasiveness and motility of A2780 cells in vitro. (A) Localization and expression of CFTR was detected by immunofluorescence staining. Cells were fixed and probed with primary antibodies against CFTR, followed by staining with fluorescein isothiocyanate-conjugated secondary antibodies and staining of nuclei with DAPI (magnification, x100). (B and C) Transwell invasion assays showed an improved invasive capacity compared with that of the control cells. "P<0.05 (magnification, $\mathrm{x} 100$ ). (D and E) Wound healing assay showing that Ad-CFTR cells had a increased migratory capacity compared with that of the control cells. "P<0.05 (magnification, $\mathrm{x} 40$ ). Values are expressed as the mean \pm standard deviation. CFTR, cystic fibrosis transmembrane conductance regulator; Ad, adenovirus.

relative width of the scratch was measured quantitatively using Adobe Photoshop 7.0 (Adobe Systems, San Jose, CA, USA). The extent of gap closure was determined as the rate of cell migration. Each assay was performed in triplicate.

Statistical analysis. Statistical analysis was performed using SPSS 17.0 software (SPSS Inc., Chicago, IL, USA). Values are expressed as the mean \pm standard deviation. A two-tailed Student's t-test assuming equal variances was performed to determine significant differences between two groups. $\mathrm{P}<0.05$ was considered to indicate a statistically significant difference between values.

\section{Results}

Construction and identification of recombinant pAd-CFTR adenoviral plasmid. The plasmid pcDNA3.1-CFTR was digested by enzymes EcoRV, SalI and KpnI, and subsequently subjected to agarose gel electrophoresis. As shown in Fig. 1A, the products included four fragments with sizes of 4.7, 2.4, 2.0 and $1.0 \mathrm{~kb}$, respectively. The fragment of $4.7 \mathrm{~kb}$ resembled CFTR and the other fragments were pcDNA3.1 fragments. The CFTR fragment from pcDNA3.1-CFTR digestion was inserted into the RFP-labeled adenoviral shuttle vector pAdTrace-TOX. Digestion of the recombinant pAdTrace-TOX-CFTR plasmid 
A

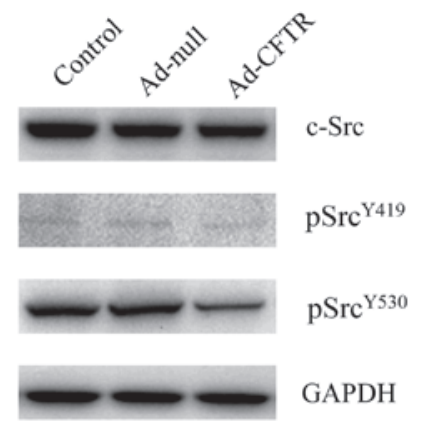

$\mathbf{B}$

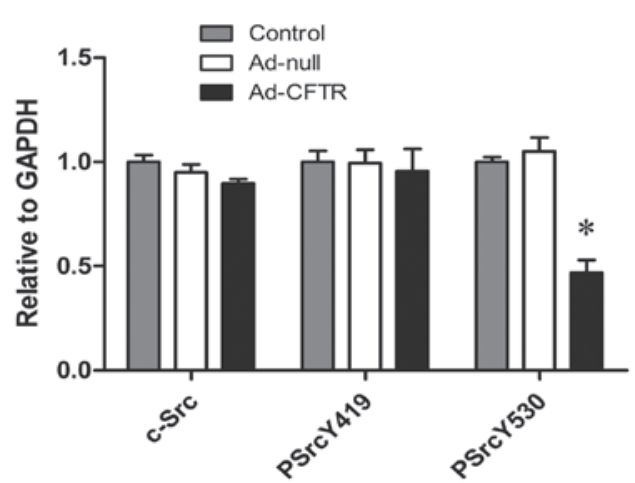

Figure 5. Ad-CFTR-mediated CFTR overexpression affects the activation of c-Src signaling. (A) A2780 cells were treated with Ad-CFTR for $48 \mathrm{~h}$ and subjected to western blot analysis for c-Src, p-Src (Tyr419) and p-Src (Tyr530). GAPDH was used as an equal loading control. (B) mRNA expression of c-Src, p-Src (Tyr419) and p-Src (Tyr530) relative to GAPDH was determined by reverse-transcription quantitative polymerase chain reaction analysis. Values are expressed as the mean \pm standard deviation of three independent experiments. $\mathrm{P}<0.05$ vs. control group. CFTR, cystic fibrosis transmembrane conductance regulator; Ad, adenovirus; p, phosphorylated.

with KpnI and EcoRV resulted in two fragments: CFTR $(4.7 \mathrm{~kb})$ and the adenoviral shuttle vector ( 9.1 kb; Fig. 1B). Sequence analysis also confirmed that the fragment inserted into pAdTrace-TOX was the human CFTR cDNA fragment obtained by PCR only. These results confirmed that the pAdTrace-TOX-CFTR containing the CFTR gene was successfully constructed. After homologous recombination with pAd-easy1 in BJ5183 E. coli cells, recombinant adenoviral vector pAd-CFTR containing the CFTR fragment was obtained and confirmed by HindIII endonuclease analysis. As shown in Fig. 1C, digestion of pAd-CFTR with HindIII produced eight fragments. Compared with the negative control (Fig. 1C; lane 2), the largest fragment of pAd-CFTR was $\sim 12.1 \mathrm{~kb}$ (Fig. 1C; lane 3) which was larger than that of pAdEasy-1 bone vector $(\sim 4,700 \mathrm{~kb})$, indicating the successful construction of the recombinant adenoviral expression vector pAd-CFTR. The target genes were confirmed to be correctly cloned into the adenoviral vector by gene sequencing and matched to the CFTR sequence in GenBank (www.ncbi.nlm. nih.gov/gene/1080).

Generation of adenoviral recombinants in HEK293 cells. The pAd-CFTR vector contains an RFP coding region driven by the CMV promoter; thus, the levels of red fluorescence are a measure of the transfection efficiency. At $24 \mathrm{~h}$ post -transfection with PacI-linearized pAd-CFTR plasmid, $>10 \%$ of HEK293 cells were RFP-positive (Fig. 2Aa and Ab). Amplification of Ad-CFTR produced a cloudy appearance of red fluorescence in the HEK293 cell line at day 10 during packaging (Fig. 2Ac and Ad). This result demonstrated that the recombinant adenoviral vector containing the human CFTR gene was successfully constructed.

Transfection of Ad-CFTR leads to overexpression of CFTR in A2780 cells. A2780 cells were transfected with Ad-CFTR and RFP-positive cells were observed by fluorescence microscopy and flow cytometry. After 48 h of transfection, $88.21 \%$ of A2780 cells were observed to be RFP-positive, while $90.15 \%$ of A2780 were RFP-positive following transfection with the empty Ad-null control vector (Figs. 2B and 3A). RT-qPCR and western blot analysis showed that native A2780 cells expressed relatively low levels of endogenous CFTR, which was not affected by transfection with Ad-null vector, while transfection with Ad-CFTR significantly induced the expression of CFTR in A2780 cells by $~ 5$-fold (Fig. 3B and C). The immunofluorescence images indicated localization of exogenous CFTR in the cytoplasm and cell membrane of A2780 cells (Fig. 4A). These results demonstrated that Ad-CFTR was able to effectively transfect A2780 cells and increase the mRNA and protein expression of CFTR.

Overexpression of CFTR enhances cell invasion and motility of A2780 cells in vitro. As shown in Fig. 4B and C, Ad-CFTR cells displayed a 1.69-fold increase in invasive capacity compared with that of the control cells. $(\mathrm{P}<0.05)$. In parallel with this, the migratory capacity of the Ad-CFTR was increased by 1.73-fold of that of the control cells $(\mathrm{P}<0.05)$ (Fig. 4D and $\mathrm{E})$.

Overexpression of CFTR affects the activation of $c$-Src signaling. To further elucidate the underlying mechanisms of CFTR-induced cell motility and invasion, the protein expression of Src kinase was evaluated in Ad-CFTR-transfected and control cells. There were no significant differences in the levels of total Src and phospho-Src (Tyr419) between any of the groups. However, Ad-CFTR cells showed decreased levels of phospho-Src (Tyr530), suggesting that CFTR may affect the invasion and motility of A2780 cells mainly through dephosphorylation of critical tyrosine residues at $530 \mathrm{bp}$ of Src (Fig. 5A and B). Further loss/gain of function studies are necessary to verify these results.

\section{Discussion}

CFTR, by controlling ion and protein transport, is thought to aid in the maintenance of cellular homeostasis in most human cells (14). Accumulating evidence suggested that CFTR has a key role in the progression and metastasis of cancer. In the female reproductive system, it has been reported that overexpression of CFTR is closely associated with the development of cervical cancer, aggressiveness of tumor cells and poor prognosis of patients (10). CFTR has a tumor-suppressing role in the development of prostate cancer, which is mediated 
via microRNA-193b targeting urokinase plasminogen activator (15). Another study indicated that CFTR expression is significantly downregulated in breast cancer, which promotes epithelial-to-mesenchymal transition and is associated with poor prognosis (16). CFTR is also known to be involved in modulating signaling pathways in cell inflammation and apoptosis $(17,18)$.

The present study constructed a vector to overexpress exogenous CFTR in SOC cells and determine its role in tumor progression. Viruses can be used for efficient gene transfer, and are able to survive and replicate in mammalian hosts (19). Adenoviral vectors are commonly used for gene transfection as the transferred DNA does not integrate into the host's genome (20). The present study successfully constructed an adenoviral vector carrying the CFTR gene by using the replication-defective AdEasy adenovirus system. Ad-CFTR was confirmed to transfect SOC cells with an efficacy of $>80 \%$, and significantly enhanced the mRNA and protein expression of CFTR. Immunofluorescence analysis indicated localization of CFTR in the cytomembrane and cytoplasm of SOC cells, suggesting that Ad-CFTR induced the expression of biologically active CFTR. Further experiments were performed to assess the effects of CFTR on the aggressiveness of ovarian cancer cells. Ad-CFTR-transfected SOC cells showed an increased cell motility and adhesion to Matrigel compared with that of the control cells. Metastasis is a complex biological process during which tumor cells acquire invasive and migratory abilities, leading to the dissemination from a primary tumor to distant secondary organs or tissues (21). Overexpression of CFTR in ovarian cancer cells enhanced their metastasis-associated migratory and invasive abilities, indicating that CFTR has a significant role in ovarian cancer metastasis and may represent a potential target for the treatment of ovarian cancer.

Studies have shown thatCFTR binds toezrin/radixin/moesin (ERM)-binding phosphoprotein of $50 \mathrm{kDa}$ (EBP50), which interacts with the ERM-family proteins via its C-terminal domain. ERM-family proteins bind to the actin cytoskeleton, which is essential in the apical polarization of CFTR in epithelial cells $(22,23)$. The present study hypothesized that the CFTR may enhance the invasive and migratory abilities of cancer cells by connecting membrane rafts to the actin cytoskeleton.

To further elucidate the underlying mechanisms of CFTR-induced cell motility and invasion, protein expression of Src kinase was evaluated in Ad-CFTR-transfected and control cells. The non-receptor tyrosine kinase Src has important roles in numerous aspects of cell physiology and is associated with signalling pathways by which cell surface receptors regulate diverse processes, including cell division, motility, adhesion, angiogenesis and survival, as well as the function of modular domains that mediate protein-protein interactions (24). Validation studies showed that Src family kinases, most notably c-Src, are frequently overexpressed and/or aberrantly activated in a variety of human cancers. Activation of c-Src is common in human ovarian cancer $(25,26)$ and is involved in ovarian cancer cell survival and resistance against chemotherapy (27). Wiener et al (28) reported that reduction in Src activity affected cellular morphology, inhibited anchorage-independent growth and diminished tumor growth in vivo. The findings of the present study indicated that upregulation of CFTR reduced the levels of phospho-Src (Tyr530), while levels of total Src and phospho-Src (Tyr419) were similar between Ad-CFTR and control groups.

The phosphorylation of the conserved tyrosine residues Tyr-416/419 and Tyr-527/530 (in chicken/human c-Src) is crucial for the regulation of c-Src, which is mostly phosphorylated at Tyr-527/530 and adopts the inactive conformation (29). The phosphorylation of Tyr-416/419 in the activation loop locks the catalytic domain in its active conformation (30). However, the conserved Tyr-527/530 in the C-terminal tail was identified as the site of inhibitory phosphorylation (31). It is known that carboxyl-terminal Src kinase (Csk), a cytoplasmic tyrosine kinase, can phosphorylate the C-terminal regulatory sites Tyr-527/530 of c-Src to inhibit its activity (29). Furthermore, Csk-binding protein ( $\mathrm{Cbp}$ ), a transmembrane protein, can recruit Csk to the membrane where c-Src is located. The Cbp-mediated re-location of Csk to the membrane may be involved in blocking Src signaling events (32). Of note, EBP50 is a specific Cbp-binding partner interacting with the $\mathrm{C}$-terminal sequence of Cbp. The Cbp-EBP50 interaction may be important for $\mathrm{Cbp}$ to recruit $\mathrm{Csk}$ to the membrane, which may facilitate the repressive function of Csk on c-Src (22). Therefore, the present study hypothesized that CFTR may indirectly affect the phosphorylation of c-Src at its Tyr-527/530 sites through interacting with EBP50, Cbp and Csk, while not affecting the Tyr-416/419 positions of Src. Furthermore, it is inferred that, as SOC cells express relatively high levels of CFTR, the induction of the progression of ovarian cancer, including migration and invasiveness, may be facilitated by de-phosphorylation of c-Src at its Tyr-527/530 sites. In line with the results of the present study, a recent study by our group performed small hairpin RNA-mediated knockdown of CFTR, which suppressed the aggressive malignant biological behavior of ovarian cancer cells in vitro and in vivo (11).

In conclusion, the present study demonstrated that adenovirus-mediated CFTR overexpression in SOC cells enhanced the migratory and invasive abilities of SOC cells in vitro. As an ion channel protein, CFTR may also be a critical candidate molecule for c-Src signal activation. Further studies on CFTR should be performed to elucidate the biochemical mechanism of the c-Src signaling pathway in SOC development.

\section{Acknowledgements}

The present study was supported by the National Natural Science Foundation of China (grant no. 81172492), the Key Project of Chongqing Science and Technology Commission (grant no. CSTC 2012JJB10030) and the Key Project of Chongqing Municipal Health Bureau (grant no. 2011-1-056).

\section{References}

1. Siegel R, Naishadham D and Jemal A: Cancer statistics, 2013. CA Cancer J Clin 63: 11-30, 2013

2. Rauh-Hain JA, Krivak TC, Del Carmen MG and Olawaiye AB: Ovarian cancer screening and early detection in the general population. Rev Obstet Gynecol 4: 15-21, 2011.

3. Lee JM, Trepel JB, Choyke P, et al: CECs and IL-8 have prognostic and predictive utility in patients with recurrent platinum-sensitive ovarian cancer: Biomarker correlates from the randomized phase-2 trial of olaparib and cediranib compared with olaparib in recurrent platinum-sensitive ovarian cancer. Front Oncol 5: 123, 2015. 
4. Chambers SK, Clouser MC, Baker AF, et al: Overexpression of tumor vascular endothelial growth factor A may portend an increased likelihood of progression in a phase II trial of bevacizumab and erlotinib in resistant ovarian cancer. Clin Cancer Res 16: 5320-5328, 2010.

5. Rankin EB, Fuh KC, Taylor TE, Krieg AJ, Musser M, Yuan J, et al: AXL is an essential factor and therapeutic target for metastatic ovarian cancer. Cancer Res 70: 7570-7579, 2010.

6. Gottesman MM, Fojo T and Bates SE: Multidrug resistance in cancer: role of ATP-dependent transporters. Nat Rev Cancer 2: 48-58, 2002

7. Higgins CF: ABC transporters: From microorganisms to man Annu Rev Cell Biol 8: 67-113, 1992.

8. Bear CE,LiCH, Kartner N, Bridges RJ,Jensen TJ, Ramjeesingh M and Riordan JR: Purification and functional reconstitution of the cystic fibrosis transmembrane conductance regulator (CFTR). Cell 68: 809-818, 1992.

9. Tizzano EF, Silver MM, Chitayat D, Benichou JC and Buchwald M: Differential cellular expression of cystic fibrosis transmembrane regulator in human reproductive tissues. Clues for the infertility in patients with cystic fibrosis. Am J Pathol 144 906-914, 1994

10. Peng X, Wu Z, Yu L, Li J, Xu W, Chan HC, Zhang Y and Hu L: Overexpression of cystic fibrosis transmembrane conductance regulator (CFTR) is associated with human cervical cancer malignancy, progression and prognosis. Gynecol Oncol 125: 470-476, 2012

11. Xu J, Yong M, Li J, Dong X, Yu T, Fu X and Hu L: High level of CFTR expression is associated with tumor aggression and knockdown of CFTR suppresses proliferation of ovarian cancer in vitro and in vivo. Oncol Rep 33, 2227-2234, 2015.

12. He TC, Zhou S, da Costa LT, Yu J, Kinzler KW and Vogelstein B: A simplified system for generating recombinant adenoviruses. Proc Natl Acad Sci USA 95: 2509-2514, 1998.

13. Livak KJ and Schmittgen TD: Analysis of relative gene expression data using real-time quantitative PCR and the 2(-Delta Delta C(T)) Method. Methods 25: 402-408, 2001.

14. Schwiebert EM, Benos DJ, Egan ME, Stutts MJ and Guggino WB CFTR is a conductance regulator as well as a chloride channel. Physiol Rev 79 (Suppl): S145-S166, 1999.

15. Xie C, Jiang XH, Zhang JT, Sun TT, Dong JD, Sanders AJ, Diao RY, Wang Y, Fok KL, Tsang LL, et al: CFTR suppresses tumor progression through miR-193b targeting urokinase plasminogen activator ( $\mathrm{UPA}$ ) in prostate cancer. Oncogene 32 : 2282-2291. $2291 \mathrm{e} 1-7,2013$.

16. Zhang JT, Jiang XH, Xie C, Cheng H, Da Dong J, Wang Y, Fok KL, Zhang XH, Sun TT, Tsang LL, et al: Downregulation of CFTR promotes epithelial-to-mesenchymal transition and is associated with poor prognosis of breast cancer. Biochim Biophys Acta 1833: 2961-2969, 2013.

17. Jacquot J, Tabary O, Le Rouzic P and Clement A: Airway epithelial cell inflammatory signalling in cystic fibrosis. Int J Biochem Cell Biol 40: 1703-1715, 2008.
18. Wu Z, Peng X, Li J, Zhang Y and Hu L: Constitutive activation of nuclear factor KB contributes to cystic fibrosis transmembrane conductance regulator expression and promotes human cervical cancer progression and poor prognosis. Int J Gynecol Cancer 23: 906-915, 2013.

19. Verma IM and Weitzman MD: Gene therapy: Twenty-first century medicine. Annu Rev Biochem 74: 711-738, 2005.

20. Björklund A, Kirik D, Rosenblad C, Georgievska B, Lundberg C and Mandel RJ: Towards a neuroprotective gene therapy for Parkinson's disease: use of adenovirus, AAV and lentivirus vectors for gene transfer of GDNF to the nigrostriatal system in the rat Parkinson model. Brain Res 886: 82-98, 2000.

21. Deryugina EI and Quigley JP: Matrix metalloproteinases and tumor metastasis. Cancer Metastasis Rev 25: 9-34, 2006.

22. Brdickova N, Brdicka T, Andera L, Spicka J, Angelisová P, Milgram SL and Horejsí V: Interaction between two adapter proteins, PAG and EBP50: A possible link between membrane rafts and actin cytoskeleton. FEBS Lett 507: 133-136, 2001.

23. Moyer BD, Denton J, Karlson KH, Reynolds D, Wang S, Mickle JE, Milewski M, Cutting GR, Guggino WB, Li M and Stanton BA: A PDZ-interacting domain in CFTR is an apical membrane polarization signal. J Clin Invest 104: 1353-1361, 1999.

24. Martin GS: The hunting of the Src. Nat Rev Mol Cell Biol 2: 467-475, 2001

25. Summy JM and Gallick GE: Src family kinases in tumor progression and metastasis. Cancer Metastasis Rev 22: 337-358, 2003.

26. Wiener JR, Windham TC, Estrella VC, Parikh NU, Thall PF, Deavers MT, Bast RC, Mills GB and Gallick GE: Activated src protein tyrosine kinase is overexpressed in late-stage human ovarian cancers. Gynecol Oncol 88: 73-79, 2003.

27. Pengetnze Y, Steed M, Roby KF, Terranova PF and Taylor CC: Src tyrosine kinase promotes survival and resistance to chemotherapeutics in a mouse ovarian cancer cell line. Biochem Biophys Res Commun 309: 377-383, 2003.

28. Wiener JR, Nakano K, Kruzelock RP, Bucana CD, Bast RCJr and Gallick GE: Decreased Src tyrosine kinase activity inhibits malignant human ovarian cancer tumor growth in a nude mouse model. Clin Cancer Res 5: 2164-2170, 1999.

29. Okada M: Regulation of the SRC family kinases by Csk. Int J Biol Sci 8: 1385-1397, 2012

30. Knighton DR, Xuong NH, Taylor SS and Sowadski JM: Crystallization studies of cAMP-dependent protein kinase. Cocrystals of the catalytic subunit with a 20 amino acid residue peptide inhibitor and MgATP diffract to 3.0 A resolution. J Mol Biol 220: 217-220, 1991 .

31. Cooper JA, Gould KL, Cartwright CA and Hunter T: Tyr527 is phosphorylated in pp60c-src: Implications for regulation. Science 231: 1431-1434, 1986.

32. Takeuchi S, Takayama Y, Ogawa A, Tamura K and Okada M: Transmembrane phosphoprotein Cbp positively regulates the activity of the carboxyl-terminal Src kinase, Csk. J Biol Chem 275: 29183-29186, 2000. 